\title{
The rise of technology in diabetes care. Not all that is new is necessarily better.
}

\author{
Abstract \\ Health-care technologies have brought many benefits to the medical profession and to \\ patients. The introduction of the continuous subcutaneous insulin infusion (CSII) pump and \\ continuous glucose monitoring (CGM) devices offers patients with Type 1 diabetes (T1D) \\ the opportunity to optimise their blood glucose control and are increasingly being \\ championed as a routine treatment approach for young people. However, the current \\ evidence base does not convincingly support arguments for the generalized application of \\ CSII and CGM into routine clinical practice. The 'patient-medical device interface' is clearly \\ a complex paradigm, and central to its success is the degree of adherence, understanding \\ and engagement demonstrated by the patient with the technology. The introduction CSII / \\ CGM technologies into the daily routine care of the patient imposes both psychological \\ and 'time-effort' burdens that many patients and families with T1D will find demanding. The \\ current application of these devices cannot therefore be considered a panacea for the self- \\ management of T1D, and raises a number of challenging problems, including those of a \\ practical, health-economic and ethical nature that need to be fully resolved before it and \\ other emerging technologies can be considered to have achieved this status.
}

\section{Introduction}

Health-care technologies have brought many benefits to the medical profession and to patients. Indeed, the rise of modern medicine has largely been founded on the introduction of innovative technologies, resulting in the more effective diagnosis and management of many medical conditions, with consequent improvements in patient quality of life and survival. However, these advancements in medical care have not been achieved without substantial cost to health-care systems and to society in general (1) (2). The increased use, and reliance, on technology has inevitably resulted in changes to how health-care is delivered, raising questions and concerns of an economic and ethical nature (3). Moreover, many of the technologies in medicine are widely used without good evidence of their safety and efficacy (4). There is also an assumption that 'high tech' equates to 'high quality' and to 'high priority' (5); a perspective that is frequently held by patients and the public, and which is often driven by a biased section of the health care profession and by an increasingly misinformed media. It is therefore not surprising that a general social myth 


\section{Pediatric Diabetes}

pervades that stipulates that 'new' is better than 'old', 'advanced' better than 'simple' and that 'more' is better than 'little' (3).

The above issues are also pertinent to the field of children and young people's diabetes, where, over the last two decades, there have been a plethora of technologies introduced into the market pace and into routine clinical practice. Prominent amongst these developments have been the introduction of the continuous subcutaneous insulin infusion (CSII) pump and continuous glucose monitoring (CGM) systems, devices that are increasingly being championed as a routine treatment approach for young people with Type 1 diabetes (T1D) by some clinicians and health-care organizations. However, the current evidence base does not convincingly support arguments for the generalized application of CSII and CGM into routine clinical practice; either from a clinical effectiveness point of view, nor from a health-economic and ethical perspective.

\section{Clinical effectiveness - the inconclusive evidence base}

Whilst diabetes related technologies such as CSII or CGM (either alone or in combination as 'sensor augmented pump' (SAP)) are now widely used in many countries, their effectiveness in clinical practice has not been consistently demonstrated, and neither is it clear which patients are most likely to benefit from it. It is generally accepted that comparisons and assessment of clinical effectiveness of medical technologies are determined on an 'evidence based' basis, relying on data from appropriately designed, and sufficiently powered, randomized controlled trials, rather from than observational studies. However such an evidence base for CSII and CGM based technologies is rather limited, particularly when studies relevant to children and adolescence with diabetes are specifically considered.

When looked at individually, most recent observational studies and published RCTs in young people appear to report either small, or modest, improvements in diabetes related outcomes, when CSII or CGM are compared against 'conventional' approaches to diabetes management with multiple daily injection (MDI) regimens (6-9) or with selfmonitoring of blood glucose (SMBG) using portable blood glucose measuring devices (10-12). However, when the data from these studies are combined and subjected to a systematic review and meta-analyses (SRMA) the same conclusions are not always supported. The SRMA is viewed as more powerful and influential in terms of evidence gathering and decision-making, with data from RCTs given priority over observational 


\section{Pediatric Diabetes}

studies. In a meta-analysis of RCTs conducted in patients with T1D (n 15) between 2002 and 2008, CSII was associated with a slightly lower glycated haemoglobin index $((\mathrm{HbA} 1 \mathrm{c})$ random-effects weighted mean difference, $0.2 \%$; 95\% confidence interval $(\mathrm{Cl}), 0.3,0.10)$, but had no significant effect on either severe or nocturnal hypoglycemia rates, when compared to MDI therapy (13). Similar findings have also been reported in a recent metaanalysis performed by Yeh and colleagues, who pooled together the data from 33 RCTs performed in children or adults with T1D; which compared CSII vs MDI (n 19), CGM vs SMBG (n 10) or SAP vs MDI + SMBG (n 4) (14). Their results showed that for patients with T1D, CSII was no different to MDI therapy in terms of glycemic control; with similar reductions in $\mathrm{HbA1c}$ levels and hypoglycaemia rates observed between the treatment groups. A separate sub-analysis of those RCTs specific to children and adolescent with T1D ( $n$ 7) did not differ in its conclusions. However, in contrast to these observations there was moderate to high strength evidence from RCTs comparing the effectiveness of CGM against SMBG to suggest that CGM technology might confer some benefit in terms of improved glycemic control (14); but this effect, was dependent on CGM adherence rates and was highest in those studies were 'sensor' adherence was sustained at levels of $60 \%$ or above (14).

Whilst comparisons of glycemic indices such as $\mathrm{HbA1c}$ and hypoglycemia rates are usually the primary outcomes of most RCTs, evaluation of patient quality of life (QoL) measures are increasingly considered to be of equal importance. It is frequently assumed that use of the latest technology is associated with greater satisfaction and contentment from a user perspective when compared to conventional treatment methods or use of older technology (3). However the evidence base derived from the diabetes setting does not convincingly support this common misconception. Low strength evidence from SRMAs suggest that CSII might confer improved QoL on patients when compared to MDI therapy, whereas CGM does not appear to have any significant impact on patient QoL when compared to standard methods of blood glucose monitoring (14).

However, it is important to acknowledge that even these SRMAs need to be interpreted with caution. Firstly, their conclusions may be already out of date given the rapidly evolving, new improved devices being introduce into the market. Secondly, SRMAs are themselves significantly limited by the poor design quality of the trials included in the analyses. The biggest problem is due to the risk of bias resulting from the lack of blinding (participants; clinicians and data assessors) and from the incomplete data and selective 


\section{Pediatric Diabetes}

outcome reporting, which is evident in most of the published trials. For these reasons doubt has been cast on whether SRMAs are relevant for clinical and economic decision making purposes as they may be significantly underestimating the utility and effectiveness of current CSII / CGM systems $(15,16)$. Alternate 'decision-making' based approaches to meta-analyses (i.e. through the pre-selection of specific clinical trials based on their intended use) have been proposed, yet even when this technique is applied the evidence in favour of CSII against conventional MDI is only modestly increased and is of weak statistical significance (16).

Observational studies, particularly those utilizing large national / multinational databases, should not be discounted. Whilst these types of studies also present a risk of bias, the data collected is invariably from over a longer period of time and is more representative of a 'typical' or 'standard' clinic population. Studies by the Hvidoere International Study Group, found no association between glycaemic control and type of insulin regimen, whether analyzed at an individual patient or by center of care level (17). Other national, multi-center, databases have reported inconsistent and contrasting observations regarding the relationship between type / mode of insulin delivery regime and outcome measures such as HbA1c or hypoglycaemia frequency (18) (19) (20). A recent combined analysis of data from 3 large national databases suggests that CSII confers improved glycaemic control compared to MDI, yet closer scrutiny of the data reveals that this result was almost entirely due to the contribution from one of the databases; representing a population with traditionally higher background mean $\mathrm{HbA1c}$ values (21). This contrasts with the data from the other two databases included in this study, where little or no difference in glycaemic control was observed between CSII and MDI patients (21). Indeed in keeping with this, other observational and RCT studies demonstrate that the greatest effective of either CSII is seen in those patients with poorest glycemic control (i.e. highest $\mathrm{HbA1c}$ or severest / most frequent hypoglycaemia) at baseline $(7,22,23)$. Furthermore, under clinical trial conditions the greatest benefit of technology use is seen in those patients who adhere strictly and engage frequently with treatment regimen requirements. CGM use, either independently or directly linked with CSII (as sensor augmented pump (SAP)), delivers its greatest benefits in terms of improved $\mathrm{HbA1c}$ and hypoglycaemia frequency in those patients who actively used CGM monitoring as instructed. Several studies document a clear relationship between improved $\mathrm{HbA} 1 \mathrm{c}$ and reduced hypoglycaemia frequency with the number of days per week of use (24) (25) (26), with greatest benefits seen in those who wear and engage with their devices for at least 60 to $70 \%$ of the time (14). However, 


\section{Pediatric Diabetes}

most observational studies reveal that less than half of young people using CGM use their device for greater than $70 \%$ of the time, and that after one year from onset $41 \%$ of patients had discontinued using it $(25,27)$. This suggests that under free-living conditions issues regarding the acceptability and utility of CGM appears to be a major hurdle limiting the effectiveness of this type of technology. This observation also emphasises the point that whatever the type of intervention, diabetes management is primarily dependent on patient behaviour and acceptability (28) and that technologies such as CSII and CGM impose an increased degree of burden and responsibility that inevitably many patients and families struggle to accept and to adhere to over the longer term. Whilst patients perceive diabetes technology use to have many positive attributes it is clear that many negative attitudes also co-exist (29-31).

\section{Cost effectiveness - the health economic uncertainties}

The costs of diabetes care have continued to rise inexorably year on year for most developed countries (32), with a substantial proportion of this attributable to patients with Type 1 diabetes and to the increasing use of technologies such as CSII and CGM. Assessment of the cost - benefit of these technologies has therefore become a priority for healthcare providers (33) and for the medical insurance industry, who increasingly are seeking best value for money. Relatively few studies have been published to date, and whilst some recent reports have predicted favourable health-economic outcomes associated with the medium to long-term use of CSII, CGM or SAP when compared to standard treatment approaches (based on a incremental cost effectiveness ratio threshold of $\$ 50,000$ per quality-adjusted life years) (34-37), others have clearly not $(38,39)$. This inconsistency in cost-effectiveness forecasting is not a surprise given the different analytical models used and the projected health-care assumptions made within them. Moreover, given the poor quality of clinical trials, and the heterogeneity of the study populations included, meaningful prediction of the cost - benefit impacts of these interventions are far from certain. Furthermore, the relevance of these health-economic analyses to children and young people with diabetes is also questionable due to the fact that the background risk factor and disease progression assumptions employed in these models are based from observations made in adult populations. Therefore the long-term cost-benefits of these technologies to young patients with T1D over other treatment approaches remains significantly in doubt, and further questions whether the current trend for increased introduction of CSII and CGM into routine practice is justified or represents 'good value for money'. 


\section{Affordability and Access - the financial and ethical constraints}

As health care costs continue to increase can we afford to pay for the widespread usage of these technologies? Despite the lack of good evidence, and because of the increasing demand by clinicians and patients, many health care organizations and providers have been compelled to produce guidance as to who should have access to these technologies $(40,41)$. Yet in most cases this guidance is too general, variably interpreted, and have 'selection' criteria that are not specific enough to identify those patients and families most likely to benefit. Furthermore many patients transitioning to the more expensive technologies may be already achieving good glycemic control with standard treatment approaches. As a result many patients starting or transferring to CSII or CGM fail to optimize their diabetes control beyond that achieved with cheaper conventional treatment regimens, or are unable to sustain initial improvements over the longer term (42) (27). Given the relatively high costs of these technologies this scenario does not represent 'good value for money' and raises a critical 'affordability' issue that our increasingly financially stretched health care systems cannot ignore. Whether accessed via publicly funded or via 'private' medical insurance based systems, diabetes technology is set to impose a significant financial burden on the individual and on society in general; contributing to heath care cost inflation and draining much needed resources from other areas of care. This financial burden will inevitably result in inequities in access to technology: with those health care providers / systems who can afford not to ration their supplies, and those individuals who have the means of paying, gaining access to these devices over and above those who cannot.

Barriers to patient access to technology also exist for other, more complex, discriminatory reasons. There is good evidence that were CSII / CGM should be readily available, that some patients who would benefit from using these devices do not access them. Clinician preference / center bias for other treatment options may be one explanation for this, but several studies have revealed that diabetes technology uptake is significantly lower in patients from certain racial/ethnic groups, and may be independently influenced by other socio-economic (e.g. medical insurance status; household income) and cultural factors (21, 43-45). Understanding the reasons for these barriers and overcoming them will be important for insuring that patients from low income and from minority groups are not discriminated against and are not denied the benefits that technology could bring to some of them. 


\section{Conclusions}

Given the current state of the art, and the evidence base available, the assertion that relatively complex and costly medical devices should be generalized to the typical patient with T1D, delivering 'superior' or 'better' outcomes over the long-term compared to conventional insulin therapy approaches, seems misplaced and unfounded. This is not to say that in the future that diabetes related technologies such as CSII and CGM (and more advanced integrated systems) will not achieve this status, as there is not doubt that technology will evolve and improve, and may even become cheaper and therefore 'costeffective', with time. Before this goal is achieved significant barriers will need to be overcome that not only improves the technical and clinical efficacy of these devices but also reduces the significant practical and psychological burdens that the current generation of devices impose. In the meantime, as we advance toward that goal, diabetes technologies such as CSII and CGM should be targeted to those patients who stand to obtain clear benefit over and above that could be achieved with conventional treatment approaches. This also means removing the discriminatory barriers that currently seems to disadvantage patients belonging to certain ethnic and socio-economic groups. As success (in glycaemic terms at least) is highly dependent on human behavior $(29,30)$, and on the degree and durability of engagement with technology over the longer term, it makes sense to develop appropriate tools and resources to rationalize the selection (and indeed deselection) of patients / families for technology use (46). Furthermore, clinicians must remain pragmatic and flexible in their approach to insulin therapy and must not loose sight of the alternate approaches available to them. New technologies must also be thoroughly and critically assessed with the same rigor as drugs are (47), and health-care workers and patients must be equally informed about the uncertainties of their benefits as well as their 'hyped' gains (3). Ultimately treatment regimens should always be individualized and diabetes care teams should be focused on achieving glycaemic targets by whatever means best suitable to the patient. 


\section{References}

1. Bodenheimer T. High and rising health care costs. Part 2: technologic innovation. Annals of internal medicine. 2005; 142:932-7.

2. Sorenson C, Drummond M, Bhuiyan Khan B. Medical technology as a key driver of rising health expenditure: disentangling the relationship. ClinicoEconomics and outcomes research : CEOR. 2013; 5:223-34.

3. Hofmann BM. Too much technology. Bmj. 2015; 350:h705.

4. Fisher ES, Welch $\mathrm{H}$. Avoiding the unintended consequences of growth in medical care: How might more be worse? JAMA. 1999; 281:446-53.

5. Fuchs VR, Sox HC, Jr. Physicians' views of the relative importance of thirty medical innovations. Health affairs (Project Hope). 2001; 20:30-42.

6. Doyle EA, Weinzimer SA, Steffen AT, Ahern JA, Vincent M, Tamborlane WV. A randomized, prospective trial comparing the efficacy of continuous subcutaneous insulin infusion with multiple daily injections using insulin glargine. Diabetes Care. 2004; 27:1554-8.

7. Opipari-Arrigan L, Fredericks EM, Burkhart N, Dale L, Hodge M, Foster C. Continuous subcutaneous insulin infusion benefits quality of life in preschool-age children with type 1 diabetes mellitus. Pediatric diabetes. 2007; 8:377-83.

8. Schiaffini R, Patera PI, Bizzarri C, Ciampalini P, Cappa M. Basal insulin supplementation in Type 1 diabetic children: a long-term comparative observational study between continuous subcutaneous insulin infusion and glargine insulin. Journal of endocrinological investigation. 2007; 30:572-7.

9. Nuboer R, Borsboom GJ, Zoethout JA, Koot HM, Bruining J. Effects of insulin pump vs. injection treatment on quality of life and impact of disease in children with type 1 diabetes mellitus in a randomized, prospective comparison. Pediatric diabetes. 2008; 9:291-6.

10. Deiss D, Bolinder J, Riveline JP, Battelino T, Bosi E, Tubiana-Rufi N, et al. Improved glycemic control in poorly controlled patients with type 1 diabetes using real-time continuous glucose monitoring. Diabetes Care. 2006; 29:2730-2.

11. Tamborlane WV, Beck RW, Bode BW, Buckingham B, Chase HP, Clemons R, et al. Continuous glucose monitoring and intensive treatment of type 1 diabetes. New England Journal of Medicine. 2008; 359:1464-76.

12. Battelino T, Phillip M, Bratina N, Nimri R, Oskarsson P, Bolinder J. Effect of continuous glucose monitoring on hypoglycemia in type 1 diabetes. Diabetes Care. 2011; 34:795-800.

13. Fatourechi MM, Kudva YC, Murad MH, Elamin MB, Tabini CC, Montori VM. Clinical review: Hypoglycemia with intensive insulin therapy: a systematic review and meta-analyses of randomized trials of continuous subcutaneous insulin infusion versus multiple daily injections. The Journal of clinical endocrinology and metabolism. 2009; 94:729-40.

14. Yeh HC, Brown TT, Maruthur N, Ranasinghe P, Berger Z, Suh YD, et al. Comparative effectiveness and safety of methods of insulin delivery and glucose monitoring for diabetes mellitus: a systematic review and meta-analysis. Annals of internal medicine. 2012; 157:336-47.

15. Price D, Graham C, Parkin CG, Peyser TA. Are Systematic Reviews and Meta-Analyses Appropriate Tools for Assessing Evolving Medical Device Technologies? Journal of diabetes science and technology. 2015.

16. Pickup JC. The evidence base for diabetes technology: appropriate and inappropriate meta-analysis. Journal of diabetes science and technology. 2013; 7:1567-74.

17. Cameron FJ, de Beaufort C, Aanstoot HJ, Hoey H, Lange K, Castano L, et al. Lessons from the Hvidoere International Study Group on childhood diabetes: Be dogmatic about outcome and flexible in approach. Pediatric diabetes. 2013; 14:473-80.

18. Holl RW, Swift PG, Mortensen HB, Lynggaard H, Hougaard P, Aanstoot HJ, et al. Insulin injection regimens and metabolic control in an international survey of adolescents with type 1 diabetes over 3 years: results from the Hvidore study group. Eur J Pediatr. 2003; 162:22-9. 19. Doggen K, Debacker N, Beckers D, Casteels K, Coeckelberghs M, Dooms L, et al. Care delivery and outcomes among Belgian children and adolescents with type 1 diabetes. European Journal of Pediatrics. 2012; 171:1679-85. 


\section{Pediatric Diabetes}

20. Maahs DM, Hermann JM, DuBose SN, Miller KM, Heidtmann B, DiMeglio LA, et al. Contrasting the clinical care and outcomes of 2,622 children with type 1 diabetes less than 6 years of age in the United States T1D Exchange and German/Austrian DPV registries. Diabetologia. 2014; 57:1578-85.

21. Sherr JL, Hermann JM, Campbell F, Foster NC, Hofer SE, Allgrove J, et al. Use of insulin pump therapy in children and adolescents with type 1 diabetes and its impact on metabolic control: comparison of results from three large, transatlantic paediatric registries. Diabetologia. 2016; 59:87-91.

22. Retnakaran R, Hochman J, DeVries JH, Hanaire-Broutin H, Heine RJ, Melki V, et al. Continuous subcutaneous insulin infusion versus multiple daily injections: the impact of baseline A1c. Diabetes Care. 2004; 27:2590-6.

23. Nimri R, Weintrob N, Benzaquen H, Ofan R, Fayman G, Phillip M. Insulin pump therapy in youth with type 1 diabetes: a retrospective paired study. Pediatrics. 2006; 117:2126-31.

24. Danne T, de Valk HW, Kracht T, Walte K, Geldmacher R, Sölter L, et al. Reducing glycaemic variability in type 1 diabetes self-management with a continuous glucose monitoring system based on wired enzyme technology. Diabetologia. 2009; 52:1496-503.

25. Weinzimer S. Prolonged use of continuous glucose monitors in children with type 1 diabetes on continuous subcutaneous insulin infusion or intensive multiple-daily injection therapy. Pediatric diabetes. 2009; 10:91-6.

26. Bergenstal RM, Tamborlane WV, Ahmann A, Buse JB, Dailey G, Davis SN, et al. Effectiveness of sensor-augmented insulin-pump therapy in type 1 diabetes. The New England journal of medicine. 2010; 363:311-20.

27. Wong JC, Foster NC, Maahs DM, Raghinaru D, Bergenstal RM, Ahmann AJ, et al. Realtime continuous glucose monitoring among participants in the T1D Exchange clinic registry. Diabetes Care. 2014; 37:2702-9.

28. Phillip M, Danne T, Shalitin S, Buckingham B, Laffel L, Tamborlane W, et al. Use of continuous glucose monitoring in children and adolescents $\left({ }^{*}\right)$. Pediatric diabetes. 2012; 13:215-28.

29. Alsaleh FM, Smith FJ, Taylor KM. Experiences of children/young people and their parents, using insulin pump therapy for the management of type 1 diabetes: qualitative review. Journal of clinical pharmacy and therapeutics. 2012; 37:140-7.

30. Ritholz MD, Smaldone A, Lee J, Castillo A, Wolpert H, Weinger K. Perceptions of psychosocial factors and the insulin pump. Diabetes Care. 2007; 30:549-54.

31. Polonsky WH, Hessler D. What are the quality of life-related benefits and losses associated with real-time continuous glucose monitoring? A survey of current users. Diabetes technology \& therapeutics. 2013; 15:295-301.

32. American Diabetes A. Economic costs of diabetes in the U.S. in 2012. Diabetes Care. 2013; 36:1033-46.

33. Cummins E, Royle P, Snaith A, Greene A, Robertson L, Mclntyre L, et al. Clinical effectiveness and cost-effectiveness of continuous subcutaneous insulin infusion for diabetes: systematic review and economic evaluation. Health Technol Assess. 2010; 14:iii-iv, xi-xvi, 1-181. 34. McQueen RB, Ellis SL, Campbell JD, Nair KV, Sullivan PW. Cost-effectiveness of continuous glucose monitoring and intensive insulin therapy for type 1 diabetes. Cost effectiveness and resource allocation : C/E. 2011; 9:13.

35. Grunberger G, Abelseth J, Bailey T, Bode B, Handelsman Y, Hellman R, et al. Consensus Statement by the American Association of Clinical Endocrinologists/American College of Endocrinology Insulin Pump Management Task Force. Endocrine Practice. 2014; 20:463-89. 36. Roze S, Saunders R, Brandt AS, de Portu S, Papo NL, Jendle J. Health-economic analysis of real-time continuous glucose monitoring in people with Type 1 diabetes. Diabetic Medicine. 2015; 32:618-26.

37. Vigersky RA. The benefits, limitations, and cost-effectiveness of advanced technologies in the management of patients with diabetes mellitus. Journal of diabetes science and technology. 2015; 9:320-30.

38. Huang ES, O'Grady M, Basu A, Winn A, John P, Lee J, et al. The cost-effectiveness of continuous glucose monitoring in type 1 diabetes. Diabetes Care. 2010; 33:1269-74. 


\section{Pediatric Diabetes}

39. Kamble S, Schulman KA, Reed SD. Cost-Effectiveness of Sensor-Augmented Pump Therapy in Adults with Type 1 Diabetes in the United States. Value in Health. 2012; 15:632-8. 40. American Diabetes A. (11) Children and adolescents. Diabetes Care. 2015; 38 Suppl:S70-6.

41. NICE. Diabetes (Type 1 and Type 2) in Children and Young People: Diagnosis and Management. Diabetes (Type 1 and Type 2) in Children and Young People: Diagnosis and Management. National Institute for Health and Care Excellence (UK)

Copyright (c) 2015 National Collaborating Centre for Women's and Children's Health., London, 2015.

42. O'Connell MA, Donath S, Cameron FJ. Poor adherence to integral daily tasks limits the efficacy of CSII in youth. Pediatric diabetes. 2011; 12:556-9.

43. Paris CA, Imperatore G, Klingensmith G, Petitti D, Rodriguez B, Anderson AM, et al. Predictors of insulin regimens and impact on outcomes in youth with type 1 diabetes: the SEARCH for Diabetes in Youth study. The Journal of pediatrics. 2009; 155:183-9.e1.

44. Icks A, Razum O, Rosenbauer J, Bachle C, Hungele A, Monkemoller K, et al. Lower frequency of insulin pump treatment in children and adolescents of Turkish background with type 1 diabetes: analysis of 21,497 patients in Germany. Diabetes technology \& therapeutics. 2012; 14:1105-9.

45. Lin MH, Connor CG, Ruedy KJ, Beck RW, Kollman C, Buckingham B, et al. Race, socioeconomic status, and treatment center are associated with insulin pump therapy in youth in the first year following diagnosis of type 1 diabetes. Diabetes technology \& therapeutics. 2013; 15:929-34.

46. Neylon OM, Skinner TC, O'Connell MA, Cameron FJ. A novel tool to predict youth who will show recommended usage of diabetes technologies. Pediatric diabetes. 2015.

47. Wilmshurst P. The regulation of medical devices. Bmj. 2011; 342:d2822. 